\title{
Availability Optimization in a Ring-Based Network Topology
}

\author{
Philippe Ezran ${ }^{\mathrm{a}, \mathrm{b}, *}$, Yoram $\operatorname{Haddad}^{\mathrm{a}}$, Mérouane Debbah ${ }^{\mathrm{b}}$ \\ ${ }^{a}$ Jerusalem College of Technology, Israel \\ ${ }^{b}$ CentraleSupélec, France
}

\begin{abstract}
Cellular networks are nowadays considered as a major critical infrastructure. Resiliency to failure due to disasters, weather based disruptions or malicious activities is essential. In the case of ring topology, because of delay and availability requirements, a wireless network connected to an aggregation node must sometimes be split into several rings. In this paper, we study the availability optimization in a ring-based network topology for a given number of cellular sites and a given size of rings. We prove that if each ring includes 3 nodes, the problem can be solved in a polynomial time, while for bigger rings, the problem is NP-hard. In this latter case, we provide approximation methods based on linear programming in order to converge to the solution.
\end{abstract}

Keywords: Cellular networks, topology, ring, availability, resiliency

\section{Introduction}

The choice of a network technology is mainly a matter of cost, availability and resiliency. For a given technology, these parameters will also impact the choice of the topology.

While fiber is capital intensive (cost function of distance) and offers limited availability, wireless is highly cost effective and flexible [1. Besides, fiber is more expensive in urban areas. The choice of fiber in urban areas may be

\footnotetext{
${ }^{*}$ Corresponding author

Email address: ezran@g.jct.ac.il (Philippe Ezran)
} 
cost-effective for very short distances only. However, this comparison must be considered cautiously: a large part of capex expenses for fiber is civil engineering. Civil engineering costs can represent up to $80 \%$ of total cost, especially in urban areas. In many cases and particularly in developing areas, these civil engineering expenses must be done anyway for infrastructure investment (road, rail, pipeline, electricity). Therefore, the relevant parameter which has to be taken into consideration for the fiber/wireless cost comparison is the additional cost generated by the fiber.

Availability is a key parameter which quantifies network performance. This parameter is closely related to reliability. The difference between these two concepts is that reliability refers to failure-free operation during an interval, while availability refers to failure-free operation at a given instant of time 2 .

Unavailability is generally defined as the sum of the unavailabilities of the network nodes [3]. According to this definition, an unavailability which impacts a great number of nodes in the network gets a higher ponderation than an unavailability which impacts a small number of nodes. However, this definition may lead to unavailabilities greater than $100 \%$. For this reason, in this paper, we define the unavailability as the percentage of time for which all or part of the network is down.

While the availability of an optical fiber connection is all or nothing, lineof-sight and propagation considerations must be taken into account in wireless links. Automatic Coding Modulation enables a microwave link to use lower modulations in degraded conditions. This difference has an impact on the network resiliency, which is the ability of the network to provide and maintain an acceptable level of service in the face of faults and challenges to normal operations [4].

In the case of a radio network, the main parameters which come into account 35 are propagation factors and infrastructural considerations. The most common topologies used in radio backhaul networks are trees and rings or a combination of both. Since tree topology generally offers shorter paths and lower costs, while ring topology generally ensures a better availability, a ring-tree combination can 
be an efficient solution to cumulate the advantages of both technologies [5].

Various causes of a network failure are identified in [4]: unusual traffic load, accidents and human mistakes, large-scale disasters, malicious attacks, environmental challenges and failures at a lower layer. A relevant network availability strategy must reduce as much as possible the failure probability of any link in the network and add redundancy in order to minimize the impact of a single

45 link failure on the availability of the network nodes.

Statistical approaches have been proposed in order to optimize availability [6], 7] for systems subject to random failures. These approaches are based upon maintenance considerations for a partially observable system.

Backhaul can be made of fiber or microwave radio. In both cases, the goal ${ }_{50}$ is to connect the base stations (BS) to the core network. In some cases, when the gateway to the core is not far, this can be performed in one hop. But in rural areas or in Ultra Dense Networks, where there are a huge number of small BS to connect, this can require multiple hops. In this paper, we consider the Microwave Radio technology as the medium to perform the backhaul. We assume that we have a large number of BS to be connected to a single aggregation node which itself will be connected to the core network. This latter connection is assumed to be wired and therefore out of the scope of this paper. Making a single large ring raises serious delay issues since the Backhaul for a BS might require several hops. In addition, it might raise serious availability issues since ${ }_{60}$ the disconnection of two links can affect a large number of BS. Therefore, it could be preferable to split the network into several rings.

In this paper, we will study the question of topology optimization from the point of view of availability maximization. Given an aggregation node and $n$ cellular sites, what is the best topology based on rings, each one of them 65 including the aggregation node, which maximizes availability? 


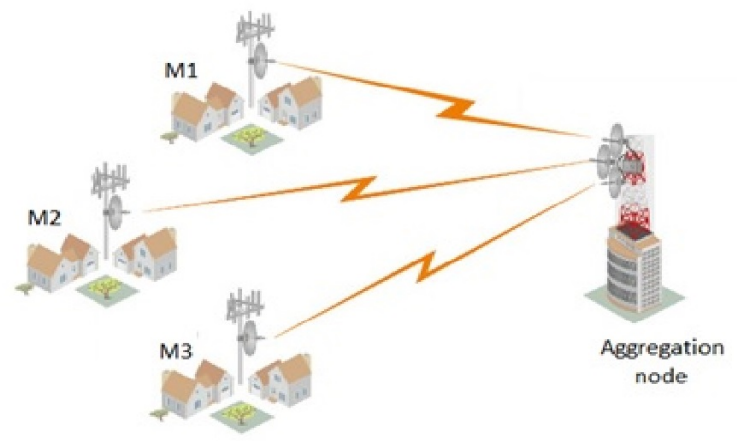

Figure 1: Backhaul network. The aggregation node handles all the traffic produced by and to nodes M1, M2, M3.

The paper is organized as follows: we first build a simplified model in Section 2 which provides a basic understanding regarding the relation between ring size and availability. In Section 3, we use existing results from graph theory in order to discuss the general model. Approximation methods based on linear programming are proposed in Section 4. Concluding remarks are given in Section 5 .

\section{Simplified model}

In a first step, we build a simplified model, based on the following five assumptions. Though the last two assumptions of this model are not realistic, this simplified approach will enable us to draw basic conclusions regarding backhaul network topologies.

Assumptions:

- the network includes $n$ cellular sites (in addition to the aggregation node);

- the network topology is made of $k$ rings;

80

- for $1 \leq i \leq k$ ring $i$ includes $n_{i}$ cellular sites and the aggregation node; $n_{1} \geq n_{2} \geq \cdots \geq n_{k} \geq 2$ 
- same failure probability for all links: $p$;

- failure events are uncorrelated.

$n$ and $n_{i}$ are related by the following equation:

$$
n=\sum_{i=1}^{k} n_{i}
$$

Availability: the condition for availability is that all cellular sites are connected to the aggregation node. This condition is fulfilled if there is no more than one failure in each ring.

$$
A=\prod_{i=1}^{k}\left((1-p)^{n_{i}+1}+\left(n_{i}+1\right) p(1-p)^{n_{i}}\right)
$$

If $p \ll 1$, this expression can be approximated by its second-order Taylor development:

$$
A=1-n \frac{p^{2}}{2}-\frac{p^{2}}{2} \sum_{i=1}^{k} n_{i}{ }^{2}+o\left(p^{2}\right)
$$

Therefore,

$$
A=1-n \frac{p^{2}}{2}-\frac{p^{2}}{2}\left(k V\left(n_{i}\right)+\frac{n^{2}}{k}\right)+o\left(p^{2}\right)
$$

where $V\left(n_{i}\right)$ is the empirical variance of the $n_{i}$ distribution:

$$
V\left(n_{i}\right)=\frac{1}{k} \sum_{i=1}^{k}\left(n_{i}-\frac{n}{k}\right)^{2}=\frac{1}{k} \sum_{i=1}^{k} n_{i}{ }^{2}-\left(\frac{n}{k}\right)^{2}
$$

Therefore, increasing the number of rings reduces the maximum path length and unavailability. On the other hand, it requires more antennas. In any case, given the number of rings, it is preferable that the empirical variance of the ring size distribution be as small as possible.

For a given number of rings $k$, the maximum availability is obtained when the empirical variance is minimized, which means when the numbers of cellular 
sites in the rings are as close as possible to $\frac{n}{k}$. Let $q$ and $r$ be the quotient and the remainder of the Euclidean division of $n$ by $k$ :

$$
n=q k+r ; 0 \leq r \leq k-1
$$

Therefore, the best availability is:

$$
\begin{gathered}
A_{k, p}=\left((1-p)^{q+2}+(q+2) p(1-p)^{q+1}\right)^{r}\left((1-p)^{q+1}+(q+1) p(1-p)^{q}\right)^{k-r} \\
A_{k, p}=1-n \frac{p^{2}}{2}-\frac{p^{2}}{2}\left(r(q+1)^{2}+(k-r) q^{2}\right)+o\left(p^{2}\right) \\
A_{k, p}=1-\frac{p^{2}}{2}\left(n+k q^{2}+2 r q+r\right)+o\left(p^{2}\right)
\end{gathered}
$$

$A_{k, p}$ is an increasing function of $k$ and a decreasing function of $p$. Of course, since the total number of antennas is $2 n+2 k$, increasing $k$ increases the cost.

The results above are illustrated with the following numerical application:

$$
\begin{aligned}
& p=0.01 \\
& n=100 \\
& 2 \leq k \leq 50
\end{aligned}
$$

The cost and the availability are growing functions of the number of rings $k$. This defines a curve of feasible. According to the price the operator is ready to pay for a given level of availability, it is possible to define an acceptable set. Any point of the feasible curve which is inside the acceptable set is a relevant choice for the operator (Fig. 2). 


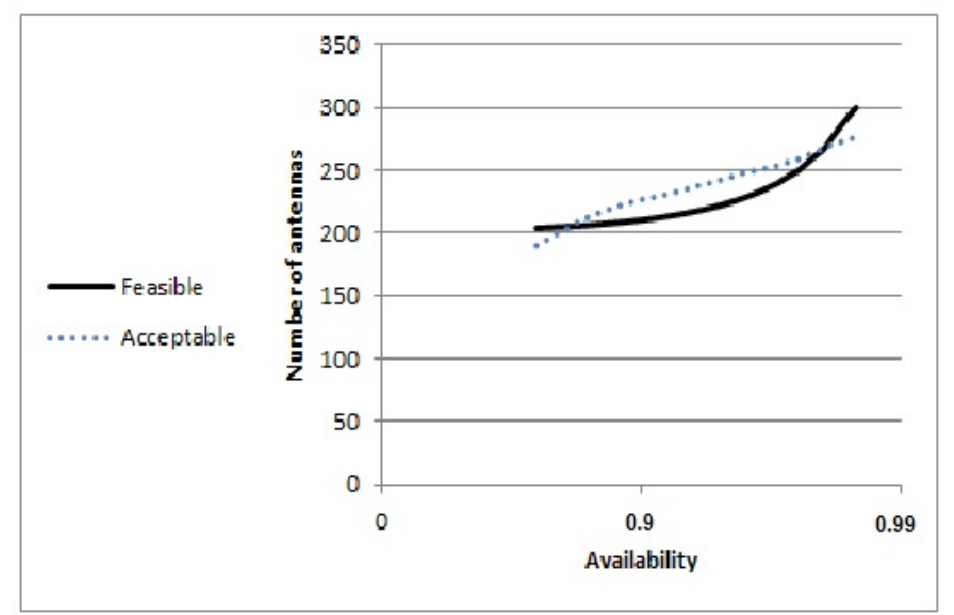

Figure 2: Cost-Availability balance.

It should be noted that this conclusion cannot be generalized to $n$ rings, each one of them including one cellular node: in this case, the network topology would be a star topology and a cellular site would be unavailable in case of single failure. The availability of a star topology network including $n$ cellular sites is:

$$
A=(1-p)^{n}=1-n p+o(p)
$$

As a consequence, availability is maximized when all the rings include 2 nodes, in addition to the aggregation node. Assuming that $n$ is even, then $k=\frac{n}{2}$ and

$$
A=\left((1-p)^{3}+3 p(1-p)^{2}\right)^{\frac{n}{2}}=\left(1-3 p^{2}+2 p^{3}\right)^{\frac{n}{2}}=1-\frac{3}{2} n p^{2}+o\left(p^{2}\right)
$$

\section{General model}

We now assume that links may have various failure probabilities. The network includes one aggregation node $(O)$ and $n$ cellular sites $M_{1}, M_{2}, \ldots, M_{n}$. 
Let $p_{i}$ be the failure probability of the link $O M_{i}$ and $p_{i j}$ the failure probanot including it.

The availability of $r$ is:

$$
\begin{gathered}
A(r)=\left(1-p_{i_{1}}\right)\left(1-p_{i_{m}}\right) \prod_{l=1}^{m-1}\left(1-p_{i_{l} i_{l+1}}\right)+p_{i_{1}}\left(1-p_{i_{m}}\right) \prod_{l=1}^{m-1}\left(1-p_{i_{l} i_{l+1}}\right)+p_{i_{m}}\left(1-p_{i_{1}}\right) \prod_{l=1}^{m-1}\left(1-p_{i_{l} i_{l+1}}\right) \\
\quad+\left(1-p_{i_{1}}\right)\left(1-p_{i_{m}}\right) \prod_{l=1}^{m-1}\left(1-p_{i_{l} i_{l+1}}\right) \sum_{j=1}^{m-1} \frac{p_{i_{j} i_{j+1}}}{1-p_{i_{j} i_{j+1}}}
\end{gathered}
$$

For a given $s \leq n$, we try to maximize the following expression:

$$
\max _{\substack{r_{1} \cup \ldots \cup r_{s}=V \\ r_{i} \cap r_{j}=\varnothing}} \prod_{i=1}^{k} A\left(r_{i}\right)
$$

Particular case. $\frac{n}{2}$ rings, each one including the aggregation node and two cel135 lular sites

In this section, we assume the following:

- $n$ is an even number;

- the network includes $\frac{n}{2}$ rings, each one including the aggregation node and 2 cellular sites;

140

- for $1 \leq i \leq n, p_{i}$ is the failure probability of the link $O M_{i}$;

- for $1 \leq i, j \leq n, p_{i j}$ is the failure probability of the link $M_{i} M_{j}$;

- failure events are uncorrelated. 
We can calculate the availability of the ring $O M_{i} M_{j}$.

$$
A_{i j}=1-p_{i} p_{j}-p_{i} p_{i j}-p_{j} p_{i j}+2 p_{i} p_{j} p_{i j}
$$

We try to maximize the expression:

$$
\prod_{\left(O, M_{i}, M_{j}\right) \in R} A_{i j}
$$

which is equivalent to maximize the expression:

$$
\sum_{\left(O, M_{i}, M_{j}\right) \in R} \log A_{i j}
$$

The problem can be regarded as a search of a perfect matching in a weighted graph: given $G=(V, E, w)$ an undirected weighted graph, the goal is to compute a perfect matching (ie a subset of edges $E^{\prime} \subseteq E$ such that each node in $V$ has exactly one incident edge in $E^{\prime}$ ) for a maximum total weight $w\left(E^{\prime}\right)$.

The maximum 2-ring division problem can be solved efficiently (in polynomial time) as followed: given a network as described above, an undirected weighted graph $G=(V, E)$ should be constructed where $V=\left\{M_{1}, M_{2}, \ldots, M_{n}\right\}$ and $E=\left(M_{i}, M_{j}\right) \mid 1 \leq i<j \leq n$ (a full graph). The weight function $w: E \rightarrow \mathbb{R}$ is defined as followed: $\forall i, j, 1 \leq i<j \leq n, w\left(M_{i}, M_{j}\right)=\log A_{i j}$. Then, due to [8], finding a maximum 2-ring division in the original network is equivalent to finding a matching $\mathcal{M}$ in $G$ such that for each matching $\mathcal{M}^{\prime}$ in $G$, $\sum_{\left(M_{i}, M_{j}\right) \in \mathcal{M}} w\left(M_{i}, M_{j}\right) \geq \sum_{\left(M_{i}, M_{j}\right) \in \mathcal{M}^{\prime}} w\left(M_{i}, M_{j}\right)$.

This problem is a well-known problem called maximum weighted matching. In 1964, Jack Edmonds was the first to develop a polynomial time algorithm to solve this problem [9]. A straight forward implementation of Edmonds' algorithm will have a running time complexity of $O\left(|V|^{2}|E|\right)$, and hence in our problem $O\left(|V|^{4}\right)$ (because the constructed graph is fully meshed. i.e. $E=\Theta\left(|V|^{2}\right)$ ). Over the years, several variants, implementations and improvements of Edmonds' idea where suggested, some of them in [8], 10, 11]. Overall, the best know algorithm for a full graph has a running time complexity of $O\left(|V|^{3}\right)$ [10, 165 [1], 12]. 
Now, solving the maximum 2-ring division problem is done in 2 phases:

1. Computing $\log A_{i j}$ for each $i, j, 1 \leq i<j \leq n$ and constructing an undirected weighted full graph $G$, as described earlier.

2. Solving the weighted maximum matching problem on $G$.

The running time complexity of phase 1 is $\left(\begin{array}{l}n \\ 2\end{array}\right) \Theta(1)+\Theta\left(n^{2}\right)=\Theta\left(n^{2}\right)$. The running time complexity of phase 2 is $O\left(n^{3}\right)$. Therefore, the running time complexity of the proposed algorithm for solving the maximum 2-ring division problem is $O\left(n^{3}\right)$. Hence, the decision problem corresponding to the maximum 2-ring division problem is in $\mathrm{P}$.

Conclusion: it is possible to connect an even number $n$ of cellular sites with $n / 2$ rings, each of one including 2 cellular sites and the aggregation node. The running time is $O\left(n^{3}\right)$.

General case. $\frac{n}{k}$ rings, each one including the aggregation node and $k$ cellular sites; $k \geq 3$

In this section, we assume the following:

- $n$ is a multiple of $k$;

- the network includes $\frac{n}{k}$ rings, each one including the aggregation node and $k$ cellular sites;

- for $1 \leq i \leq n, p_{i}$ is the failure probability of the link $O M_{i}$;

- for $1 \leq i, j \leq n, p_{i j}$ is the failure probability of the link $M_{i} M_{j}$;

- failure events are uncorrelated.

At first, we investigate the relation between the general maximum $k$-ring division problem and an NP-Complete problem.

Let $\mathcal{P}_{k}(n)$ be the set of $k$-combinations of $\{1,2, \ldots, n\}$. Given a family of sets $F \subseteq \mathcal{P}_{k}(n)$ for $k \geq 3$, a $k$-set packing of $\{1,2, \ldots, n\}$ is a set $S \subseteq F$ such that $\forall s_{1}, s_{2} \in S, s_{1} \cap s_{2}=\varnothing$. The maximum $k$-set packing problem (MSP) is to find a $k$-set packing $S$ of $\{1,2, \ldots, n\}$ such that for each $k$-set packing $S^{\prime}$ of 
$\{1,2, \ldots, n\},|S| \geq\left|S^{\prime}\right|$. The corresponding decision problem $(d-M S P)$ is a well-known NP-Complete problem [13, [14].

We define the maximum production $[0,1)$ weighted $k$-set packing (MPWSP) as followed: given a family $F=\mathcal{P}_{k}(n)$ where $n=m k$ for some $m \in \mathbb{N}$ and a weight function $w: F \rightarrow[0,1)$, the MPWSP problem is to find a $k$-set packing $S$ of $\{1,2, \ldots, n\}$ such that for each $k$-set packing $S^{\prime}$ of $F, \prod_{u \in S} w(u) \geq \prod_{u \in S^{\prime}} w(u)$.

Let $d-M P W S P$ denote the corresponding decision problem to MPWSP. $d-M S P$ is a particular case of $d-M P W S P$ with:

- $w(X)=1$ for $X \in F$

- $w(X)=0$ for $X \notin F$

Therefore, $d-M P W S P$ is as least as hard as $d-M S P$. Thus, d-MPWSP is NP-Hard (and in fact, d-MPWSP is NP-Complete).

Given an algorithm to solve the MPWSP problem, it can be used to solve the general maximum $k$-ring division problem as followed: let $A_{i_{1} i_{2} \ldots i_{k}}^{\max }$ be the highest availability of all the rings including the $k$ nodes $i_{1}, i_{2}, \ldots, i_{k}$ and the aggregation node:

$A_{i_{1} i_{2} \ldots i_{k}}^{\max }=\max \left(A_{j_{1} j_{2} \ldots j_{k}} \mid j_{1} j_{2} \ldots j_{k}\right.$ is a permutation of $\left.i_{1} i_{2} \ldots i_{k}\right)$

and

$$
c_{i_{1} i_{2} \ldots i_{k}}=\log \left(A_{i_{1} i_{2} \ldots i_{k}}^{\max }\right)
$$

An instance of MPWSP could be constructed by defining a family of sets $F=\mathcal{P}_{k}(n)$ and a weight function $w\left(i_{1}, i_{2}, i_{k}\right)=A_{i_{1} i_{2} \ldots i_{k}}^{\max }$. Clearly, a solution to the constructed MPWSP instance yields a solution to the original maximum $k$-ring division problem. 
Reciprocally, let us consider the following particular case:

$$
\begin{aligned}
& V=U_{1} \cup \ldots \cup U_{k} \\
& \left|U_{1}\right|=\ldots=\left|U_{k}\right|=\frac{n}{k} \\
& \forall u \in U_{1}, p_{u}=0 \\
& \forall u \in U_{2} \cup \ldots \cup U_{k}, p_{u}=1 \\
& \forall u_{i} \in U_{i}, \forall u_{j} \in U_{j},|j-i| \neq 1 \rightarrow p_{i j}=1 \\
& \forall u_{i} \in U_{i}, \forall u_{j} \in U_{j},|j-i|=1 \rightarrow p_{i j} \in[0,1]
\end{aligned}
$$
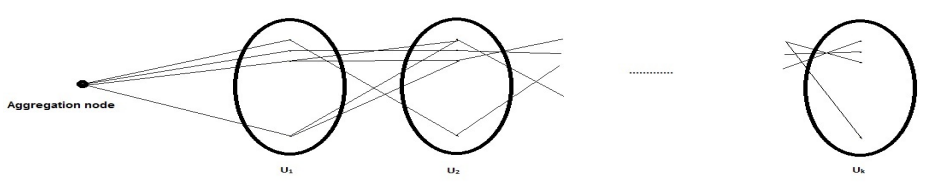

Figure 3: Particular case of $k$-ring division.

In this particular case, the aggregation node is connected to all the nodes of $U_{1}$ and no other node. Every connection between the aggregation node and anyone of the nodes of $U_{1}$ is assumed to be free of failure-risk. A node in a given subset $U_{i}$ can be connected only to the nodes belonging to the adjacent sets $U_{i-1}$ and $U_{i+1}$.

Then, the maximization of $c_{i_{1} i_{2} \ldots i_{k}}$ is a $k$-dimensional matching problem, which is known to be NP hard [15] for $k \geq 3$. Therefore, the general problem is NP hard for $k \geq 3$.

\section{Approximation methods}

Since the general maximum k-ring division problem is NP-hard for $k \geq 3$, we propose hereafter approximation methods in order to converge to the solution. 
We can present the k-ring division problem as an ILP: the idea is to define binary variables which correspond to a $k$-ring.

$$
P=\max \sum_{\left\{i_{1}, i_{2}, \ldots, i_{k}\right\} \in \mathcal{P}_{k}(n)} c_{i_{1} i_{2} \ldots i_{k}} x_{i_{1} i_{2} \ldots i_{k}}
$$

subject to

$$
\begin{gathered}
\sum_{\substack{\left\{i_{1}, i_{2}, \ldots, i_{k}\right\} \in \mathcal{P}_{k}(n) \\
j \in\left\{i_{1}, i_{2}, \ldots, i_{k}\right\}}} x_{i_{1} i_{2} \ldots i_{k}}=1, \forall j \in\{1,2, \ldots, n\} \\
x_{i_{1} i_{2} \ldots i_{k}} \in\{0,1\}, \forall\left\{i_{1}, i_{2}, \ldots, i_{k}\right\} \in \mathcal{P}_{k}(n)
\end{gathered}
$$

The purpose of this method is to characterize the network topology by binary values: $x_{i_{1} i_{2} \ldots i_{k}}=1$ if the nodes $i_{1}, i_{2}, \ldots, i_{k}$, together with the aggregation node, form a ring, and $x_{i_{1} i_{2} \ldots i_{k}}=0$ else.

Constraints 19 and 20 forces each node $j$ to be in exactly one k-ring.

General ILP is known to be NP-Hard [12. However, linear programming can be solved in polynomial time. By replacing constraint (20) in (18) with the constraint:

$$
x_{i_{1} i_{2} \ldots i_{k}} \geq 0, \forall\left\{i_{1}, i_{2}, \ldots, i_{k}\right\} \in \mathcal{P}_{k}(n)
$$

(also known as LP relaxation) we get a polynomial-time solvable linear program.

Without loss of generality, we can assume that $c_{i_{1} i_{2} \ldots i_{k}}>0$ for each $\left\{i_{1}, i_{2}, \ldots, i_{k}\right\} \in \mathcal{P}_{k}(n)$, since we can always add any constant to all the coeffi235 cients $c_{i_{1} i_{2} \ldots i_{k}}$. Doing that does not change the set of vectors that maximizes the problem, because due to the constraints, each feasible vector contains exactly $\frac{n}{k}$ ones and $\left(\begin{array}{l}n \\ k\end{array}\right)-\frac{n}{k}$ zeros. Therefore, adding $K$ to all the coefficients $c_{i_{1} i_{2} \ldots i_{k}}$ is equivalent to adding the constant $K \frac{n}{k}$ to the original objective function. 
Lemma 4.1. Assuming $c_{i_{1} i_{2} \ldots i_{k}}>0$ for each $\left\{i_{1}, i_{2}, \ldots, i_{k}\right\} \in \mathcal{P}_{k}(n)$, a vector $x$ which maximizes the system

$$
P^{\prime}=\max \sum_{\left\{i_{1}, i_{2}, \ldots, i_{k}\right\} \in \mathcal{P}_{k}(n)} c_{i_{1} i_{2} \ldots i_{k}} x_{i_{1} i_{2} \ldots i_{k}}
$$

subject to

$$
\begin{gathered}
\sum_{\substack{\left\{i_{1}, i_{2}, \ldots, i_{k}\right\} \in \mathcal{P}_{k}(n) \\
j \in\left\{i_{1}, i_{2}, \ldots, i_{k}\right\}}} x_{i_{1} i_{2} \ldots i_{k}} \leq 1, \forall j \in\{1,2, \ldots, n\} \\
x_{i_{1} i_{2} \ldots i_{k}} \in\{0,1\}, \forall\left\{i_{1}, i_{2}, \ldots, i_{k}\right\} \in \mathcal{P}_{k}(n)
\end{gathered}
$$

is feasible to (18).

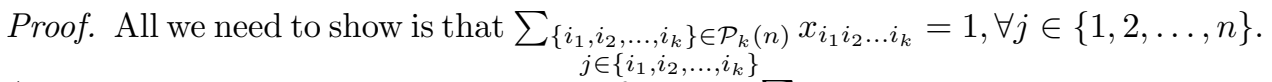
Assume by contradiction that there is a $j$ for which $\sum_{\substack{\left\{i_{1}, i_{2}, \ldots, i_{k}\right\} \in \mathcal{P}_{k}(n) \\ j \in\left\{i_{1}, i_{2}, \ldots, i_{k}\right\}}} x_{i_{1} i_{2} \ldots i_{k}}=0$ (there is no other possibility since $x$ satisfies constraint (24); this means that there is a node $j$ that is not in any $k$-ring). Since $n$ is a multiplier of $k$, there are $k-1$ other nodes that are not in any $k$-ring, therefore, a new ring can be added to the sum contradicting the fact that $x$ maximizes $P^{\prime}$.

Lemma 4.2. Assuming $c_{i_{1} i_{2} \ldots i_{k}}>0$ for each $\left\{i_{1}, i_{2}, \ldots, i_{k}\right\} \in \mathcal{P}_{k}(n)$, a vector $x$ which maximizes (22) maximizes (18).

Proof. Straight from Lemma 4.1) and from the fact that any feasible vector in (18) is a feasible vector in 22 .

However, not each solution to the relaxation yields a solution to the original problem. Consider the following example:

$$
\begin{gathered}
n=6 ; k=3 \\
c_{124}=c_{135}=c_{236}=c_{456}=1 ; \text { all other } c_{i j l}=0
\end{gathered}
$$

Then, $\max \sum c_{i j l} x_{i j l}$ subject to [19), 21] is obtained only for:

$$
x_{124}=x_{135}=x_{236}=x_{456}=\frac{1}{2} ; \text { all other } x_{i j l}=0
$$




\subsection{Power method}

In order to favour the emergence of a maximum which has exclusively integer

255 penalize potential non-integer solutions.

For each $\alpha>0$ we define:

$$
P_{\alpha}=\max \sum_{\left\{i_{1}, i_{2}, \ldots, i_{k}\right\} \in \mathcal{P}_{k}(n)} c_{i_{1} i_{2} \ldots i_{k}} x_{i_{1} i_{2} \ldots i_{k}}^{\alpha}
$$

subject to

$$
\begin{gathered}
\sum_{\substack{\left\{i_{1}, i_{2}, \ldots, i_{k}\right\} \in \mathcal{P}_{k}(n) \\
j \in\left\{i_{1}, i_{2}, \ldots, i_{k}\right\}}} x_{i_{1} i_{2} \ldots i_{k}} \leq 1, \forall j \in\{1,2, \ldots, n\} \\
x_{i_{1} i_{2} \ldots i_{k}} \geq 0, \forall\left\{i_{1}, i_{2}, \ldots, i_{k}\right\} \in \mathcal{P}_{k}(n)
\end{gathered}
$$

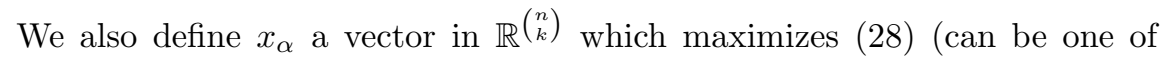
many if there is more than one vector which maximizes $P_{\alpha}$ ).

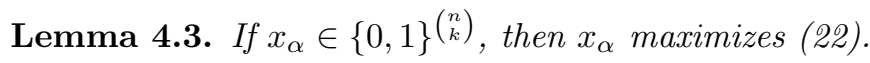

Proof. $x_{\alpha}$ is obviously feasible to $(22)$. Assume by contradiction that there is $y$ which satisfies (23) and 24) for which $P^{\prime}(y)>P^{\prime}\left(x_{\alpha}\right)$. Since all coordinates of $x_{\alpha}$ and $y$ are 0 or $1, P^{\prime}\left(x_{\alpha}\right)=P_{\alpha}\left(x_{\alpha}\right)$ and $P^{\prime}(y)=P_{\alpha}(y)$. Therefore, $P_{\alpha}(y)>P_{\alpha}\left(x_{\alpha}\right)$, contradicting the fact that $x_{\alpha}$ maximizes 28 .

Lemma 4.4. $\lim _{\alpha \rightarrow \infty} P_{\alpha}\left(x_{\alpha}\right)=\lim _{\alpha \rightarrow \infty} P_{\alpha}\left(\left\lfloor x_{\alpha}\right\rfloor\right)$

Proof.

$$
\begin{aligned}
& \lim _{\alpha \rightarrow \infty} P_{\alpha}\left(x_{\alpha}\right)=\lim _{\alpha \rightarrow \infty} \sum_{x_{i_{1} i_{2} \ldots i_{k}}} \sum_{\text {coordinates of } x_{\alpha}} c_{i_{1} i_{2} \ldots i_{k}} x_{i_{1} i_{2} \ldots i_{k}}^{\alpha} \\
& =\lim _{\alpha \rightarrow \infty} \sum_{x_{i_{1} i_{2} \ldots i_{k}}} \sum_{\text {coordinates of } x_{\alpha}} c_{i_{1} i_{2} \ldots i_{k}}\left\lfloor x_{i_{1} i_{2} \ldots i_{k}}\right\rfloor^{\alpha}=\lim _{\alpha \rightarrow \infty} P_{\alpha}\left(\left\lfloor x_{\alpha}\right\rfloor\right)
\end{aligned}
$$

The second equality derives from the fact that constraints 29$)$ and 30 force that $0 \leq x_{i_{1} i_{2} \ldots i_{k}} \leq 1$. Hence: 
- if $x_{i_{1} i_{2} \ldots i_{k}}=1$, then $c_{i_{1} i_{2} \ldots i_{k}} x_{i_{1} i_{2} \ldots i_{k}}^{\alpha}=c_{i_{1} i_{2} \ldots i_{k}}\left\lfloor x_{i_{1} i_{2} \ldots i_{k}}\right\rfloor^{\alpha}=c_{i_{1} i_{2} \ldots i_{k}}$

Theorem 4.5. Assuming $c_{i_{1} i_{2} \ldots i_{k}}>0$ for each $\left\{i_{1}, i_{2}, \ldots, i_{k}\right\} \in \mathcal{P}_{k}(n)$, as $\alpha \rightarrow \infty,\left\lfloor x_{\alpha}\right\rfloor$ maximizes $[18)$.

Proof. From lemma (4.4), we conclude that $\left\lfloor x_{\alpha}\right\rfloor$ maximizes (28). Then, from lemma (4.3), we conclude that $\left\lfloor x_{\alpha}\right\rfloor$ maximizes $(22)$. Finally, from lemma 4.2 , we conclude that $\left\lfloor x_{\alpha}\right\rfloor$ maximizes 18$]$.

Theorem 4.5 offers an alternative way to solve (18) for a large $\alpha$ and select the floor values of the elements in $x_{\alpha}$.

\section{Conclusion}

Availability is maximized when the number of rings is high and the ring size distribution is regular. In this paper, we show that the partition of a network including an aggregation node and $n$ cellular sites into $\frac{n}{2}$ rings, each one including the aggregation node and 2 cellular sites, can be solved in a time of $O\left(n^{3}\right)$. Regarding a partition with larger rings, the problem is similar to a $k$-set partition problem, which is NP-hard for $k \geq 3$. We propose an approximation method, based on linear programming and use of an exponent aimed to accelerate the convergence.

\section{Acknowledgments}

The authors would like to thank Yosef Pogrow for his highly valuable contribution to this work and to acknowledge the contribution of the COST action TD1207. The authors alone are responsible for the content.

This research was funded by the Office of the Chief Scientist of the Israel Ministry of Economy under the Neptune generic research project. Neptune is the Israeli consortium for network programming. 


\section{References}

[1] Microwave Backhauling - Meeting the Capacity Challenges of 4G/LTE and Beyond, Ceragon Technical Brief (2012) 1-10.

[2] M. Al-Kuwaiti, N. Kyriakopoulos, S. Hussein, A comparative analysis of network dependability, fault-tolerance, reliability, security, and survivability, IEEE Communications Surveys \& Tutorials 11.2 (2009) 106-124.

[3] H. Lehpamer, Microwave Transmission Networks, McGraw-Hill, 2010.

[4] J. P. Sterbenz, D. Hutchison, E. K. Çetinkaya, A. Jabbar, J. P. Rohrer, M. Schller, P. Smith, Resilience and survivability in communication networks: Strategies, principles, and survey of disciplines, Computer Networks 54.8 (2010) 1245-1265.

[5] R. Nadiv, T. Naveh, Wireless Backhaul Topologies: Analysing Backhaul Topology Strategies, Ceragon White Paper (2010) 1-15.

[6] I. B. Gertsbakh, Statistical Reliability Theory, CRC Press, 1988.

[7] R. Jiang, M. J. Kim, V. Makis, Availability maximization under partial observations, OR spectrum 35 (3) (2013) 691-710.

[8] H. N. Gabow, Implementation of algorithms for maximum matching on nonbipartite graphs, Ph.D. thesis (1974).

[9] J. Edmonds, Maximum matching and a polyhedron with 0,1 vertices, J. Res. Nat. Bur. Standards B 69.196569 (125-130) (1965) 55-56.

[10] H. N. Gabow, An efficient implementation of Edmonds' algorithm for maximum matching on graphs, Journal of the ACM (JACM) 23.2 (1976) 221234.

[11] H. N. Gabow, Data structures for weighted matching and nearest common ancestors with linking, University of Colorado, Boulder, Department of Computer Science, 1990. 
[12] A. Schrijver, Theory of linear and integer programming, Wiley, 1998.

[13] V. Kolmogorov, Blossom V: a new implementation of a minimum cost perfect matching algorithm, Mathematical Programming Computation 1.1 (2009) 43-47.

325 [14] E. Hazan, S. Safra, O. Schwartz, On the complexity of approximating k-set packing, computational complexity 15.1 (2006) 20-39.

[15] S. Polyakovskiy, F. C. Spieksma, G. J. Woeginger, The three-dimensional matching problem in Kalmanson matrices, Journal of Combinatorial Optimization 26.1 (1) (2013) 1-9. 